\title{
Benign Germinative Follicular Epithelium Neoplasm
}

National Cancer Institute

\section{Source}

National Cancer Institute. Benign Germinative Follicular Epithelium Neoplasm. NCI

Thesaurus. Code C43319.

A benign neoplasm involving the germinative follicular epithelium. 